\title{
Mixing of binary fluids with composition-dependent viscosity in a T-shaped micro-device
}

\author{
Chiara Galletti ${ }^{1}$, Giacomo Arcolini, Elisabetta Brunazzi, Roberto \\ Mauri \\ Department of Civil and Industrial Engineering, Laboratory of Multiphase Reactive Flows, \\ Università di Pisa, I-56126 Pisa, Italy
}

\begin{abstract}
The process of laminar mixing in a T-shaped micro-device is studied by direct numerical simulation for a model binary mixture, composed of two fluids having the same density and the same viscosity, yet presenting a strong fluidity of mixing effect, i.e. the viscosity of the mixture is a function of its composition. In all cases, the inlet streams remain separated up to a critical Reynolds number, corresponding to the transition from a vortex flow regime, with a double mirror symmetry, to an engulfment flow regime, with a point central symmetry. In the case of a positive fluidity of mixing, the onset of the engulfment regime is accompanied by a sharp increase of the degree of mixing, with the critical Re decreasing as the fluidity of mixing increases. On the contrary, when the fluid mixture has a larger viscosity than that of its pure components, a viscous layer forms at the confluence of the inlet flows, which tends to keep the two streams separated. Therefore, in this case, no sudden increase of the degree of mixing is observed at the onset of the engulfment regime.
\end{abstract}

Keywords: Micromixing; Multiphase flow; Fluidity of mixing; Laminar flow.

\section{Introduction}

In a recent work, Orsi et al. (2013a) simulated the mixing process that follows the confluence of two fluids onto a T-junction, comparing the case where the two inlet fluids are both water with the case where one inlet fluid is water and the other is ethanol. In particular, it was shown that the degree of mixing increases sharply when the flow field turns from vortex to engulfment flow regimes, that is from a mirror symmetric morphology that keeps the two streams separated, to a point symmetric pattern, where fluid elements reach

\footnotetext{
${ }^{1}$ Author to whom correspondence should be addressed. Electronic mail: chiara.galletti@unipi.it.
}

Preprint submitted to Chemical Engineering Science 
the opposite side of the mixing channels. Orsi et al. (2013a) observed that the symmetry breaking process, corresponding to the onset of the engulfment regime, occurs at $R e \approx 140$ in the water-water case, in agreement with several works in literature (see Engler et al., 2004; Bothe et al., 2006; Hoffmann et al. 2006; Galletti et al., 2012), whereas larger $R e$ numbers (i.e., $R e=230$ ) were needed to enhance mixing in the water-ethanol case. The reason of this mixing hindrance was ascribed to the fact that a water-ethanol mixture has a viscosity that is almost three times larger than that of water, so that at the confluence of the T-mixer, the two streams are separated by a viscous interfacial layer that hampers vortex formation and retards mixing.

Viscosity change due to mixing, however, is not the only possible cause of the above mentioned mixing hindrance. First of all, we should consider that the two pure fluids have different viscosities, i.e., ethanol is about $20 \%$ more viscous than water. This, however, by increasing the ethanol residence time, should enhance mixing. Then, there is the effect of density differences, which usually enhance fluid separation. In fact, the density of a water-ethanol mixture is a strong function of its composition: water is about $20 \%$ heavier than ethanol and, in addition, the volume of a water-ethanol mixture may be up to $5 \%$ smaller than the sum of the volumes that are initially occupied by its components. This latter, so-called volume of mixing, effect was studied in a separate work by Orsi \& Mauri (2013), showing that a volume sink at the confluence of the two fluid streams induces a velocity field heading towards the interface, but this effect is small and cannot be the cause of the above-mentioned suppression of mixing efficiency. As for the different densities of water and ethanol, Orsi et al. (2013a) concluded that this effect is important only at low Reynolds numbers, when the two fluids segregate vertically, but does not affect the strong variation of the degree of mixing that was observed. This point, however, deserves further investigations.

In this work, we want to corroborate the statement that the main cause of mixing reduction is the increase of the mixture viscosity upon mixing. To do that, we focus on the effect of the concentration-dependent viscosity, by totally eliminating any density changes and viscosity offset. Therefore, we consider a model binary mixture which, on one hand, has a constant density, while, on the other hand, has a viscosity that strongly depends on composition, although the two pure fluids have the same viscosity.

Our model of a composition-dependent mixture viscosity mirrors the thermodynamic description of a composition-dependent mixture volume. In fact, from multicomponent thermodynamics, we know that the density $\rho$ (i.e., the inverse of the specific volume) of a binary mixture is related to the densities $\rho_{A}$ and $\rho_{B}$ of pure fluids $A$ and $B$, respectively, through the following relation:

$$
\frac{1}{\rho}=\frac{\phi}{\rho_{A}}+\frac{1-\phi}{\rho_{B}}+\Delta v_{m i x},
$$

where $\phi$ is the mass fraction of component $A$. Here, we see that the difference between the specific volume of the mixture and the sum of the volumes of the pure components at constant temperature and pressure is the volume of mixing, 
$\Delta v_{\text {mix }}$, whose value is a function of the mixture composition. In particular, so-called regular mixtures are characterized by $\Delta v_{m i x}=0$, so that their volume is conserved. In this work, as mentioned above, we assume that $\rho_{A}=\rho_{B}=\rho_{0}$ and $\Delta v_{m i x}=0$, so that $\rho=\rho_{0}$.

Many different mixing rules can be found in the literature to evaluate the viscosity of a mixture starting from that of its pure components (see Laliberté, 2007). Considering that viscosity represents the resistance of a fluid against the diffusive transport of momentum, and that in fluid mixtures these resistances are in parallel (Ottino \& Chella, 1983), the viscosity $\mu$ of a binary mixture can be related to the viscosities of pure fluids $A$ and $B, \mu_{A}$ and $\mu_{B}$, respectively, through the following relation, which is similar to the density one:

$$
\frac{1}{\mu}=\frac{\phi}{\mu_{A}}+\frac{1-\phi}{\mu_{B}}+\Delta f_{m i x}
$$

where $\Delta f_{m i x}$ is the fluidity of mixing, that accounts for the non-ideality of the mixture. This term is particularly important; for example, a mixture of ethanol and water at $20^{\circ} \mathrm{C}$ with $0.3<\phi<0.6$ has a viscosity which is almost three times that of pure water (Simmonds, 1919). Similar behavior is observed for many aqueous mixtures of organic solvents, such as acetone, methanol, propanol and acetic acid (see Dizechi \& Marschall, 1982; Wang et al., 2004; Laliberté, 2007). On the other hand, the fluidity of mixing can also be positive, as for example in the cases of 1,2-dichloroethane/carbon-tetrachloride (Zhang \& Han, 1997; Mahajan \& Mirgane, 2013; Gonzalez et al., 2007).

Here, since our objective is to investigate the effect of the fluidity of mixing $\Delta f_{m i x}$ alone, we eliminate any viscosity offset by assuming that the two pure fluids have the same viscosity, i.e. $\mu_{A}=\mu_{B}=\mu_{0}$. In addition, we assume for the viscosity of the mixture the following simple, quadratic expression:

$$
\mu(\phi)=\mu_{0}[1+4(\alpha-1) \phi(1-\phi)],
$$

where $\alpha$ expresses the ratio between the viscosity of a $50 \%-50 \%$ mixture and that of a pure fluid species. Three cases will be considered, namely $\alpha=3$, $\alpha=\frac{1}{3}$ and $\alpha=1$. The former, $\alpha=3$ case, presents a negative fluidity of mixing and corresponds, indicatively, to a water-ethanol mixture, where the effects due to density changes, together with those due to the different viscosities of the two pure species, have been eliminated. The second case, with $\alpha=\frac{1}{3}$, presents a positive fluidity of mixing and corresponds approximately to octan-2-ol/ntetradecane(Mahajan \& Mirgane, 2013); finally, the $\alpha=1$ case, corresponds to two identical fluids.

In the present work, we will determine both velocity and concentration fields resulting from the confluence of the model fluid mixtures at a T-junction.

\section{Problem description}

\subsection{The governing equations}

Consider two fluids at the same temperature and density, converging onto a $\mathrm{T}$ junction. Assuming that the heat of mixing and the volume of mixing are neg- 
ligible, so that the process can be modeled to be isothermal and isovolumetric, at steady state the governing equations are:

$$
\begin{gathered}
\rho_{0} \mathbf{v} \cdot \nabla \mathbf{v}+\nabla P=\nabla \cdot\left[\mu(\phi)\left(\nabla \mathbf{v}+(\nabla \mathbf{v})^{T}\right)\right], \\
\nabla \cdot \mathbf{v}=0 \\
\mathbf{v} \cdot \nabla \phi=D \nabla^{2} \phi
\end{gathered}
$$

where $\mathbf{v}$ is the solenoidal velocity field, $P$ is the dynamic pressure, $\phi$ the mass (and volume) fraction of, say, component $A$ of the binary mixture, and $D$ is the molecular diffusivity, while the $T$ superscript indicates the transpose of a tensor. If the two fluids are identical, we can imagine adding a very small amount of contaminant, i.e. a dye, to one of the fluids (which therefore continue to have the same physical properties) and therefore, in this case, $\phi$ indicates the (normalized) dye mass fraction. As mentioned in the Introduction, the characteristics of the velocity and concentration fields can be described through the Reynolds and Peclet numbers,

$$
R e=\frac{\rho_{0} U d}{\mu_{0}} ; \quad P e=\frac{U d}{D}=R e S c ; \quad S c=\frac{\mu_{0}}{\rho_{0} D},
$$

where $S c$ is the Schmidt number, $U$ is the mean velocity, while the characteristic fluid length $d$ is assumed to be the hydraulic diameter, i.e.,

$$
d=\frac{2 W H}{W+H},
$$

where $W$ and $H$ are the mixing channel width and height, respectively (see Figure 1).

Finally, as mentioned above, we assume that the process is isothermal, therefore neglecting the temperature change due to the heat of mixing. As shown in Orsi et al. (2013a) in the water-ethanol case, this hypothesis is verified, as the effect of the heat of mixing consists of a small temperature decrease along the mixing channel, which amounts to a negligible change in the properties (i.e. density and viscosity) of the mixture.

\subsection{Geometry and numerical model}

The geometric setting of our simulation consists of the T-shaped micro device shown in Figure ??. The mixing channel has a rectangular cross section with a 2:1 aspect ratio (i.e. width $W=2 H$ and depth $H$ ), with length $L=15 d$, while the inlet channels are identical, with square cross section (i.e. width $W_{i}=H$ and depth $H$ ) and length $L_{i}=L=2.25 d$. Such length $L_{i}$ was chosen to include the whole confluence region, while the length of the mixing channel, $L$, was long enough to completely describe the complex vortical structures and avoid any channel-length effect on the predictions. In particular, using $H=100 \mu m$, we obtain the geometric setup used by many investigators (see, for example, the numerical work by Bothe et al., 2006, and the experimental work by Hoffmann 


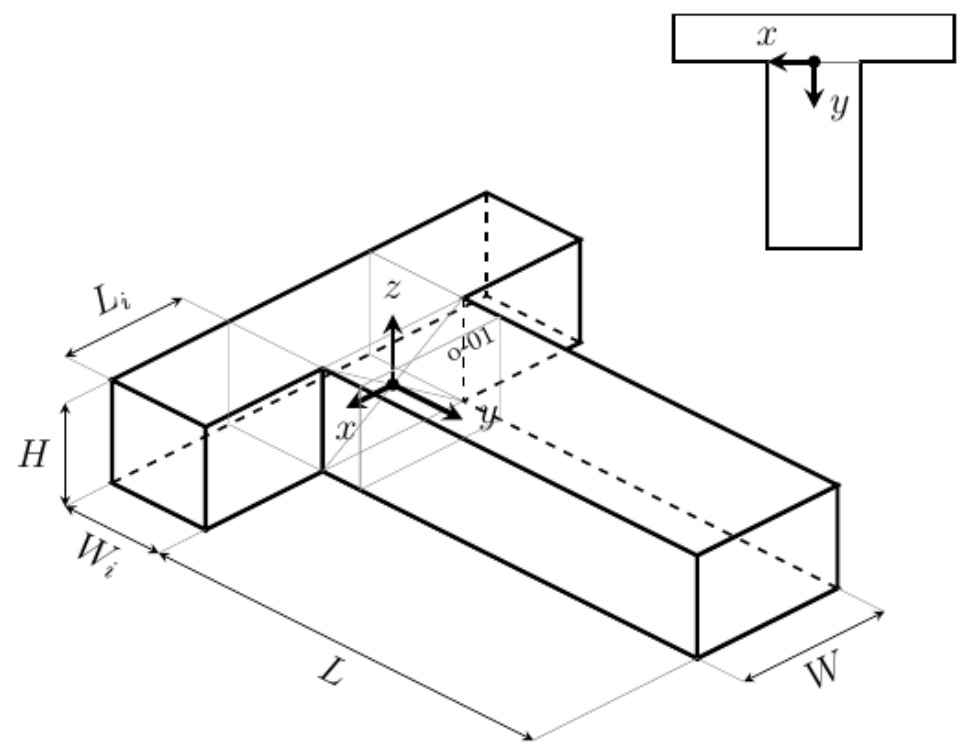

Figure 1: Sketch of the T mixer. $o-01$ section corresponds to $y=1.5 d$

et al., 2006). The reference system, whose origin is placed at the entrance of the mixing channel, is shown in Figure 1. In particular, $y$ denotes the distance from the confluence of the inlet streams.

Simulations were performed using the commercial software Ansys 14.5, with the FLUENT fluid dynamics package, that we used as a Navier-Stokes solver, where dimensions and velocities were scaled in terms of the hydraulic diameter $d$ and the mean velocity $U$, i.e. $y *=y / d$ and $u *=u / U$. The grid was obtained from a grid independency study and consists of cubic elements with $H / 20$ edge, leading to $20 \times 40$ elements in each cross section of the mixing channel, thus in agreement with the recommendation by Hussong et al. (2009), regarding how to accurately describe the velocity fields. A second order discretization scheme was used to solve all the governing equations. Simulations were typically considered converging when the normalized residuals for velocities were stationary with iterations and fell below $1 \times 10^{-12}$. Such small residuals were specifically required to ensure converged solutions near the engulfment, as shown by Galletti et al. (2012). The steadiness of the solution with iterations was also assessed by checking the velocity and concentration field in the outlet section of the mixing channels at different iterations.

As mentioned above, here we discretize the concentration field using the same mesh size as for the velocity field. Clearly, since in our case $S c \gg 1$, so that $P e \gg R e$, a rigorous simulation of mass diffusion would require a grid resolution that is much finer than that of the velocity field (see the extensive discussion in Ottino, 1994, about this issue). This, however, would lead to unacceptably long computation times, which can be resolved only by using clever numerical schemes, such as the one suggested by Bothe et al., 2011). 
Adopting, as we do here, the same mesh size, effectively amounts to studying mass diffusion on a coarse-grained length-scale. Clearly, this approximation is unacceptable when mixing is a diffusion-driven process, as it happens when, in the $\alpha=1$ case at $R e \ll 1$, the two inlet steams flow side by side, so that a concentration boundary layer of thickness $\delta \sim d P e^{-1 / 2}$ forms at the interface. However, in our case, as $R e>100$, convection at the confluence occurs also along a transverse direction, i.e., perpendicularly to the mean flow, as each inlet flow penetrates into the other by a typical distance, $R$ (as we saw, $R \cong d$ in the engulfment regime). This is particularly true when viscosity depends on composition, since a transverse flow is induced by the term $(d \mu / d \phi) \nabla \phi \cdot \nabla \mathbf{u}$ appearing in Eq. (4). Accordingly, defining a transverse Peclet number, $P e_{t}=$ $U R / D$, we see that in all cases $P e_{t} \gg 1$, indicating that purely diffusive fluxes can be neglected compared to the convective fluxes induced by transverse fluid motion, so that an approximately correct concentration field can be determined by assuming that the fluid particles are passive tracers and that we can follow their motion in the coarse-grained scale of the velocity field. In the appendix of Orsi et al. (2013a) this statement is supported by the results of a perturbation analysis, showing that when $S c \gg 1$, at leading order the diffusive term of the convective-diffusive Eq. (6) can be neglected. Here, this conclusion will be further reinforced showing that the concentration field and the degree of mixing do not depend on the $S c$ number.

The boundary conditions consisted of no-slip velocity and no-mass-flux at the channel walls, a constant ambient pressure at the exit, while at the two entrances a given flow profile was imposed, assuming fully developed flow conditions. In this way, we avoid the undesired complications connected to the inlet fluid conditions, that we have analyzed in a separate work (Galletti et al., 2012). The equation for the fully developed profile in a rectangular conduit can be found in Happer \& Brenner (1965) and Chatwin \& Sullivan (1982) as explained in Galletti et al. (2012). The flow rates of the two fluids were equal for all the cases studied.

\subsection{Degree of Mixing}

The definition of mixing efficiency is based on mass fluxes, and it is similar to that reported in Orsi et al. (2013a). Accordingly, as density here is uniform, we can define the degree of mixing in a cross section of the outlet channel as:

$$
\delta_{m}(y)=1-\frac{\sigma_{b}(y)}{\sigma_{\max }} ; \quad \sigma_{\max }=\sqrt{\bar{\phi}_{b}\left(1-\bar{\phi}_{b}\right)} .
$$

where $\sigma_{b}$ is the standard deviation of the volumetric flow, with

$$
\sigma_{b}^{2}(y)=\frac{\int_{S}\left(\phi(x, y, z)-\bar{\phi}_{b}\right)^{2} u(x, y, z) d x d z}{\int_{S} u(x, y, z) d x d z}=\frac{1}{N \bar{u}} \sum_{i}\left(\phi_{i}-\bar{\phi}_{b}\right)^{2} u_{i},
$$

In equations 9 and 10, $\sigma_{\max }$ denotes the maximum value of the standard deviation $\sigma_{b}$, that is achieved when the two streams remain completely segregated, while $\overline{\phi_{b}}$ is the "bulk" mass fraction: 


$$
\overline{\phi_{b}}=\frac{\dot{V}_{A}}{\dot{V}}=\frac{\int_{S} \phi(x, y, z) u(x, y, z) d x d z}{\int_{S} u(x, y, z) d x d z}=\frac{\sum_{i} \phi_{i} u_{i}}{N \bar{u}},
$$

where $u_{i}$ is the axial velocity of the mixture at point $i$ within the cross section of the mixing channel, while $\bar{u}$ is its mean value, with:

$$
\dot{V}=\bar{u} S ; \quad \bar{u}=\frac{1}{S} \int_{S} u(x, y, z) d x d z .
$$

\section{Results}

\subsection{Model validation: $\alpha=1$}

First of all, we considered the $\alpha=1$ case, corresponding to a mixture of two liquids having the same viscosity (and density). As mentioned above, that represents the case of two identical liquids, where a very dilute solute has been dissolved into one of the inlet streams, so that $\phi$ represents the normalized mass fraction of the solute. In Figure 2 the projections of the velocity vectors on the cross section in the mixing channel at a distance $y=1.5 d$ from the confluence are represented for different $R e$. We see that when $R e<138$ the flow morphology is characterized by two pairs of counter-rotating vortical structures, originating at the confluence of the two inlet streams. This flow field, characterised by a double mirror symmetry, has been observed both experimentally and numerically (see Engler et al., 2004; Hoffmann et al., 2006; Bothe et al., 2006) and is denoted as vortex flow regime.

Figure 3a shows the distribution of normal vorticity for $R e=125$ at different cross sections of the mixing channel, namely at distances $y=0.1 d, 0.8 d$ and $1.5 d$ from the confluence of the inlet streams. The gray region represents the vortical structures as identified through the $\lambda_{2}$ criterion (Jeong \& Hussain, 1995). Such criterion is based on the concept that a vortex is associated to a local pressure minimum. Accordingly, a vortex is defined as a connected fluid region where the second eigenvalue of the symmetric tensor $\mathbf{S}^{2}+\boldsymbol{\Omega}^{2}$ is negative, i.e. $\lambda_{2}<0$, with $\mathbf{S}$ and $\boldsymbol{\Omega}$ indicating the strain rate and vorticity tensors, respectively, i.e. $\nabla \mathbf{v}=\mathbf{S}+\boldsymbol{\Omega}$.

It is well evident the double mirror symmetry of the flow field with a double pair of counter-rotating vortical structures, whose legs are of the same length and parallel to the mixing channel. For that reason the vortex flow is also denoted as steady symmetric regime in Fani et al. (2013).

Then, at $R e=138$, a symmetry breaking is observed, with a transition from vortex to engulfment flow regime, that is from double mirror to central point symmetry structures, where the latter are invariant under point reflection through the center of the cross section. This is well evident from Figure $2 \mathrm{~d}$ which represents the in-plane vectors at the mixing channel cross section located at $y=1.5 d$, for $R e=150$. The vortical structures of Figure 3c clearly indicate the presence of two stronger legs, extending into the mixing channel, together with other two weaker legs. Such flow structures are very similar to the ones 


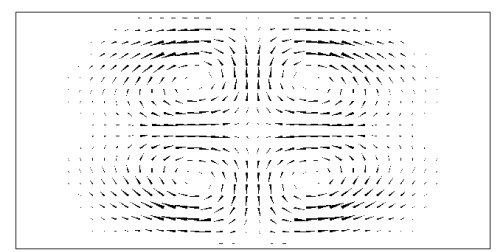

(a) $\operatorname{Re}=112$

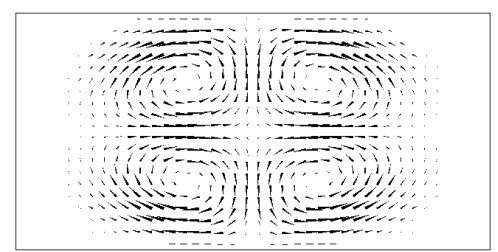

(c) $\operatorname{Re}=137$

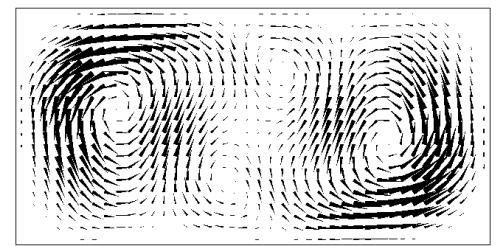

(e) $\operatorname{Re}=160$

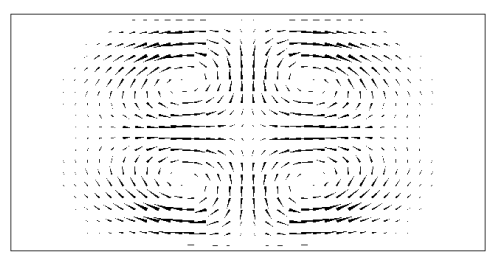

(b) $\operatorname{Re}=125$

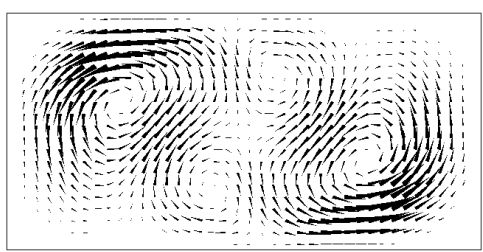

(d) $\operatorname{Re}=150$

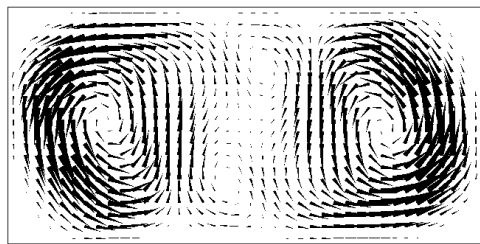

(f) $\operatorname{Re}=175$

Figure 2: Vector plots in the mixing channel cross section at $y=1.5 d$ for $\alpha=1$ at different Re numbers 


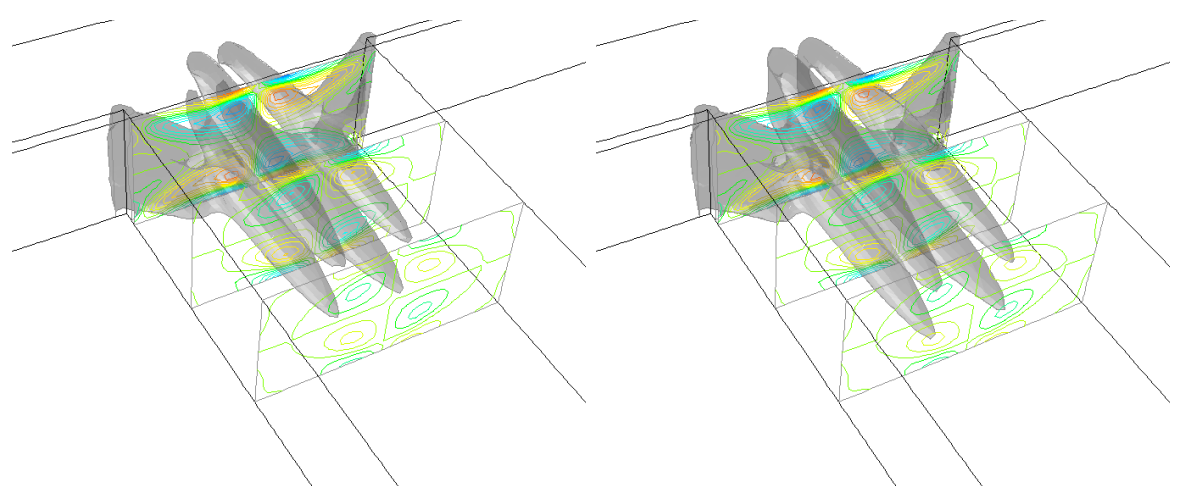

(a) $\operatorname{Re}=125$

(b) $\operatorname{Re}=137$

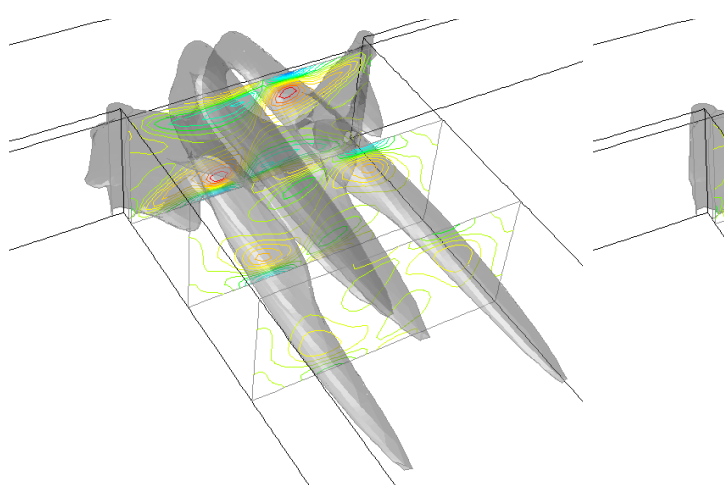

(c) $\operatorname{Re}=150$

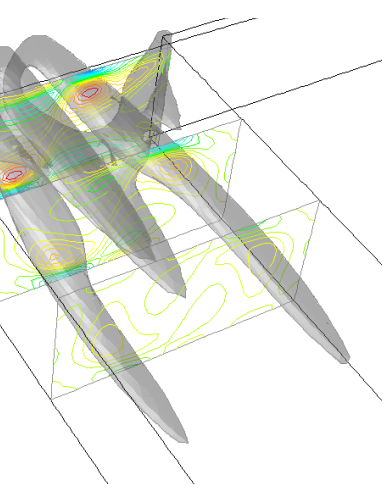

(d) $\operatorname{Re}=160$

Figure 3: Identified vortices according to the $\lambda_{2}$ criterion and distribution of normal vorticity for $\alpha=1$ and different $R e$ numbers at planes: $y=0.1 d, 0.8 d$ and $1.5 d$.

observed by Fani et al. (2013), who used a T-mixer with a different aspect ratio. The engulfment flow regime is also denoted as steady asymmetric regime.

The transition between the two flow regimes is particularly evident in Figure 4 , where the concentration field at the outlet of the mixing channel (i.e. at $y=15 d)$ is represented for different $R e$. We see that in the vortex regime, when $R e<138$, the two inlet streams basically remain segregated from each other, while they mix very effectively in the engulfment regime.

\subsection{Role of molecular diffusivity}

In most liquid-liquid mixing processes, molecular diffusivity is much smaller than kinematic viscosity, and, in fact, in our simulation we assumed that the Schmidt number is $S c=10^{4}$. As we mentioned in the Introduction, though, when, as in our case, $P e \gg R e$, a rigorous simulation of mass diffusion would require a grid resolution that is much finer than that of the velocity field. Here, on the contrary, we discretize the concentration field using the same mesh size as the velocity field, which effectively amounts to studying mass diffusion on 
a coarse-grained length-scale. In this respect, the degree of mixing that we obtain at low $R e$ is overestimated; however, since its value is very small, we may conclude that this effect is negligible. The same conclusion was reached by repeating the simulations for $R e=100$ and $R e=150$ (i.e. both for vortex and engulfment regimes), assuming decreasing values of $S c$. In fact, representing the degree of mixing, $\delta_{m}$, as a function of the inverse Schmidt number, $S c^{-1}$, in Figure 5 we see that, when $S c>10^{3}, \delta_{m}$ tends to a constant value, which essentially corresponds to the passive tracer limit. The same result was reached by Orsi et al. (2013a), who applied the homogenization (i.e., an asymptotic expansion) technique to show that at leading order the diffusion term in the convection-diffusion equation can be neglected, provided that the unperturbed velocity field is not perpendicular to the mass diffusive flux.

As we have previously mentioned, the results of the $\alpha=1$ case are in perfect agreement with previous investigations, thus validating our numerical scheme. Therefore, the mixing process when $\alpha=1$ will be considered as a base case, against which the other two cases (namely, $\alpha=3$ and $\alpha=\frac{1}{3}$ ) will be compared.

\subsection{Negative fluidity of mixing: $\alpha=3$}

In Figure 6 we present the in-plane velocity vectors at $y=1.5 d$ and for different $R e$ of a fluid mixture with $\alpha=3$, i.e., whose viscosity is larger than that of its pure components. At lower $R e$, we observe a double mirror symmetric vortex regime (see Figure 6 a at $R e=112$ ), with similar vortical structures as for the $\alpha=1$ case. With increasing $R e$, the double mirror symmetry disappears, but that happens gradually, as can be seen from Figure 6. This is confirmed by the vortical structures shown in Figure 7, that exhibit legs of almost the same length, although the double-mirror symmetry transforms gradually into a central point symmetry. We can say that there is no more a sudden transition from the vortex to the engulfment regime.

The concentration distribution at the outlet of the mixing channel is shown in Figure 8 for different $R e$ numbers. Here, we see two additional features that characterize the mixing process of a mixture with a negative fluidity of mixing. First, the two inlet streams start to mix even at values of the Reynolds number as low as $R e=125$, due to the transversal convection induced by the viscosity gradient, while in the base case, such transversal flow field being absent, the two streams remain sharply separated. This result is in agreement with the observation by Govindarajan \& Sahu (2014) that the convection induced by viscosity gradients strongly regularizes the flow field, thus hampering any instability. The other important feature is that a viscous layer, separating the two streams, is present even in the engulfment regime, so that the degree of mixing for $\alpha=3$ remains lower than that for $\alpha=1$. Such viscous layer can be identified as the green region in the figures, corresponding to a $50 \%-50 \%$ mixture. For that reason, even though the engulfment regime (defined here as the loss of the double mirror symmetry of the flow field) starts at lower $R e$ numbers, the contours of Figure 8 indicate that larger $R e$ numbers are needed to significantly improve mixing. This is in agreement with the results by Orsi et al. (2013a, 2013b), who observed a significant decrease of the degree of mixing 
for a water-ethanol mixture occurring at a larger Reynolds number, compared with the water-water case.

\subsection{Positive fluidity of mixing: $\alpha=\frac{1}{3}$}

The behaviour of fluid mixtures with $\alpha=\frac{1}{3}$, i.e., whose viscosity is smaller than that of its pure components, is quite different from the previous two cases, as it is evident from the velocity vectors at $y=1.5 d$ shown in Figure 9 . Here, the loss of symmetry can be observed for $R e$ as low as $R e=118$ and it is rather sudden, as can be seen from the two strong vortices depicted in Figure 9c at $R e=125$.

The complex vortical structures are shown in Figure 10, where we see that at $R e=118$ the double mirror symmetry has disappeared. With increasing $R e$, we observe the growth of two weaker vortices near the axis of the mixing channel, that are coupled to stronger, swirling vortical structures located externally. Moreover, we observe an additional pair of vortical structures originating from the T-mixer corners, which merge with the main vortices in the mixing channel, thus further increasing their vorticity (see Figures 10c and 10d). Then, at $R e=175$, the simulations with the steady solver did not converge and transient simulations should be adopted instead, indicating that for such $R e$ the flow becomes unsteady. Hence, the onset of the unsteady regime is largely anticipated with respect to the constant viscosity base case where, for this T-mixer geometry, the transition occurs at $R e \approx 240$ (Bothe et al., 2006; Dreher et al., 2009). The behaviour of the vortical structures in the unsteady regime has been discussed by Fani et al. (2014), using a T-mixer with a different aspect ratio.

The concentration distribution at the mixing channel outlet is shown in Figure 11, revealing that the mixing process is much more effective than in the $\alpha=3$ case. In addition, we see that the two inlet streams start to mix even at very low values of the Reynolds number, due to the transversal convection induced by the viscosity gradient. Since the same anticipated mixing occurs for $\alpha=3$ as well, we conclude that this behavior characterizes all mixtures with a composition-dependent viscosity, i.e. either presenting a non-zero fluidity of mixing (positive or negative), or being composed of two fluids with different viscosities.

\subsection{Comparison between different cases}

The degree of mixing evaluated at the channel outlet is compared for all cases in Figure 12 as a function of Re. The single fluid case $(\alpha=1)$ indicates the well known sudden increase of the degree of mixing occurring at $R e \approx 138$ due to the onset of the engulfment regime. In fact, from $R e=138$ to $R e=175$, $\delta_{m}$ increases from 0 to $52 \%$.

Mixtures with a positive fluidity of mixing $\left(\alpha=\frac{1}{3}\right)$ exhibit also a sudden increase of mixing, coinciding with the onset of the engulfment regime, occuring at $R e \approx 118$. Here, from $R e=118$ to $R e=160, \delta_{m}$ increases from 0 to $77 \%$.

Mixtures with a negative fluidity of mixing $(\alpha=3)$, unlike the other cases, show a gradual increase of the degree of mixing, reaching $\delta_{m}=34 \%$ when 
$R e=175$. We observe that at $R e=125$, the degree of mixing is larger than that of the $\alpha=1$ base case, because of the positive effect on mixing of the viscosity gradients mentioned above. As Re increases, though, the degree of mixing is lower than that of the $\alpha=1$ base case, because of the presence of a separating viscous layers, that hampers the development of a strong engulfment flow.

It is also worth noting that in the vortex flow regime (see $R e=112$ ) the degree of mixing is very small for $\alpha=1$, while it is different from zero, albeit still small, in the presence of a positive or negative fluidity of mixing, due to the transversal convective flow induced by viscosity gradients.

Finally, the degree of mixing along the mixing channel for all cases and different Reynolds numbers is reported in Figure 13. Here, we observe that at $R e=125$ and $R e=137$, the degree of mixing of both non regular mixtures is larger than that of the $\alpha=1$ base case, whereas for larger Re numbers, the mixture with a negative fluidity $(\alpha=3)$ provides the smallest mixing efficiency. Moreover, the degree of mixing along the channel reaches almost the outlet value at $y \approx 7.5 d$ for $\alpha=1$ and $\alpha=3$, whereas it continues to increase for $\alpha=\frac{1}{3}$.

\section{Conclusions and discussion}

Laminar mixing of non-regular binary mixtures is studied numerically, considering the effect on the velocity field of the viscosity change of the two fluids as they are brought in contact with one another. A model mixture is considered, consisting of two fluids having the same density, $\rho_{0}$, and the same viscosity, $\mu_{0}$, yet presenting a strong fluidity of mixing effect, i.e., the viscosity of the mixture is a quadratic function of its composition, reaching a maximum value, $\mu_{\max }$, at a $50 \%-50 \%$ fluid composition. In particular, we consider three cases, where either $\alpha=\mu_{\max } / \mu_{0}=3$ (negative fluidity of mixing), $\alpha=1$ (zero fluidity of mixing) or $\alpha=1 / 3$ (positive fluidity of mixing). These cases refer to mixtures whose viscosity increases, remains constant or decreases upon mixing, respectively.

First, the numerical approach was validated showing that, in the constantviscosity case, well known results are obtained. In addition, we show that, although here the Schmidt number $S c \gg 1$, i.e. molecular diffusivity is much smaller than kinematic viscosity, we may adopt the same mesh size to study the fluid velocity and concentration fields, provided that the transversal Peclet, $P e_{t}$, number is large. In fact, when $P e_{t} \gg 1$, purely diffusive fluxes can be neglected compared to the convective fluxes induced by transverse fluid motion, so that an approximately correct concentration field can be determined by assuming that the fluid particles are passive tracers and that we can follow their motion in the coarse-grained scale of the velocity field. In fact, representing the degree of mixing, $\delta_{m}$, as a function of $S c$, we show that, when $S c \gg 1, \delta_{m}$ tends to a constant value, which essentially corresponds to the passive tracer limit. The same result was reached by Orsi et al. (2013a), who applied an asymptotic expansion technique to show that at leading order the diffusion term in the 
convection-diffusion equation can be neglected, provided that the unperturbed velocity field is not perpendicular to the mass diffusive flux.

In the $\alpha=1$ base case we observe that the inlet streams remain separated up to a critical Reynolds number, $R e \approx 138$, corresponding to a transition from the vortex flow regime, with a double mirror symmetry, to the engulfment flow regime, with a point central symmetry. The critical Reynolds number corresponds also to a sharp increase of the degree of mixing.

Such a critical Re number is found to decrease for positive fluidity of mixing, i.e., $R e_{c} \approx 118$ when $\alpha=\frac{1}{3}$. In this case, the vortical structures in the engulfment regime are complex, exhibiting swirling legs of the main vortices in the mixing channel. As a consequence, the onset of the unsteady regime was found to be largely anticipated as well at $R e=175$, compared to $R e \approx 240$ in the $\alpha=1$ base case (Dreher, 2009).

In the case of a negative fluidity of mixing $(\alpha=3)$, a completely different behaviour is observed, with a smooth transition from the vortex to the engulfment regime. On one hand, as in the positive fluidity of mixing case, the perfect double mirror symmetry is lost at lower $R e$ compared to the $\alpha=1$ base case, thanks to the forces induced by viscosity gradients. However, here as the inlet fluids are put in contact with one another, they form a separating viscous layer that hampers mixing and that continues to be observed even in the engulfment flow regime. This viscous layer retards the diffusion of one fluid into the other, thereby limiting the degree of mixing, which at $R e=175$ is approximately $30 \%$ lower than that of the $\alpha=1$ base case.

\section{Acknowledgment}

This work was supported in part by the Italian Ministry of Education and Research (MIUR), grant PRIN, No. 2009-3JPM5Z. 


\section{References}

Bothe, D., Stemich, C., Warnecke, H.J., 2006. Fluid mixing in a T-shaped micro-mixer. Chem. Eng. Sci. 61, 2950-2958.

Bothe, D., Lojewski, A., Warnecke, H.J., 2011. Fully resolved numerical simulation of reactive mixing in a T-shaped micromixer using parabolized species equations. Chem. Eng. Sci. 66, 6424-6440.

Chatwin, P.C., Sullivan, P.J., 1982. The effect of aspect ratio on the longitudinal diffusivity in rectangular channels. J. Fluid Mech. 120, 347358.

Dizechi, M., Marschall, E., 1982. Viscosity of some binary and ternary liquid mixtures, J. Chem. Eng. Data 27, 358-363.

Dreher, S., Kockmann, N., Woias, P. 2009. Characterization of laminar transient flow regimes and mixing in T-shaped micromixers. Heat Trans. Eng. 30, 91-100.

Engler, M., Kockmann, N., Kiefer, T., Woias, P., 2004. Numerical and experimental investigations on liquid mixing in static micromixers. Chem. Eng. J. 101, 315-322.

Fani, A., Camarri, S., Salvetti, M.V., 2013. Investigation of the steady engulfment regime in a three-dimensional T-mixer. Phys. Fluids 25, 064102.

Fani, A., Camarri, S., Salvetti, M.V., 2014. Unsteady asymmetric engulfment regime in a T-mixer. Phys. Fluids 26, 074101.

Galletti, C., Roudgar, M., Brunazzi, E., Mauri,R., 2012. Effect of inlet conditions on the engulfment pattern in a T-shaped micro-mixer. Chem. Eng. J. 185-186, 300-313.

González, B., Calvar, N., Gómez, E., Domínguez, A., 2007. Density, dynamic viscosity, and derived properties of binary mixtures of methanol or ethanol with water, ethyl acetate, and methyl acetate at $\mathrm{T}=293.15$, 298.15, and 303.15 K, J. Chem. Thermodyn. 39, 1578 - 1588.

Govindarajan, R., Sahu, K.C., 2014. Instabilities in viscosity-stratified flow, Annu. Rev. Fluid Mech. 46, 331-353.

Happel, J., Brenner, H., 1965. Low Reynolds number hydrodynamics, Prentice Hall, Englewood Cliffs, NJ, Eq. (2-5.24).

Hoffmann, M., Schlüter, M., Räbiger, N., 2006. Experimental investigation of liquid-liquid mixing in T-shaped micro-mixers using $\mu$-LIF and $\mu$-PIV. Chem. Eng. Sci. 61, 2968-2976. 
Hussong, J., Lindken, R., Pourquie, M., Westerweel, J., 2009. Numerical study on the flow physics of a T-shaped micro-mixer, IUTAM bookseries 15, 191-205.

Jain, P., Singh, M., 2004. Density, viscosity, and excess properties of binary liquid mixtures of propylene carbonate with polar and nonpolar solvents, J. Chem. and Eng. Data 49, 1214-1217.

Jeong, J., Hussain F., 1995. On the identification of a vortex. J. Fluid Mech. 285, 69-94.

Laliberté, M., 2007. Model for calculating the viscosity of aqueous solutions, J. Chem. and Eng. Data 52, 321-335.

Mahajan, A.R., Mirgane, S.R., 2013. Excess molar volumes and viscosities for the binary mixtures of n-octane, n-decane, n-dodecane, and ntetradecane with octan-2-ol at 298.15K, J. Thermodyn. 2013, 571918.

Orsi, G., Roudgar, M., Brunazzi, E., Galletti, C., Mauri, R., 2013a. Water-ethanol mixing in T-shaped microdevices, Chem. Eng. Sci. 95, 174-183.

Orsi, G., Galletti, C., Brunazzi, E., Mauri, R., 2013b. Mixing of two miscible liquids in T-shaped microdevices, Chem. Eng. Trans. 32, 14711476 .

Orsi, G., Mauri, R., 2013. Volume of mixing effect on fluid counterdiffusion, Phys. Fluids 25, 082101.

Ottino, J.M., 1994. Mixing and chemical reactions a tutorial, Chem. Eng. Sci. 49, 4005 - 4027.

Ottino, J.M., Chella, R., 1983. Laminar mixing of polymeric liquids; a brief review and recent theoretical developments, Polym. Eng. \& Science $23,357-379$.

Simmonds, C., 1919. Alcohol, Its Production, Properties, Chemistry, and Industrial Applications, Macmillan, NewYork

Wang, P., Anderko, A., Young, R.D., 2004. Modeling viscosity of concentrated and mixed-solvent electrolyte systems, Fluid Phase Equil. 226, 71-82.

Zhang, H.-L., Ha, S.-J., 1997. Viscometric and volumetric studies on binary mixtures of 1,2-dichloroethane and chlorinated methanes or their binary equimolar mixtures at 303.15 K, Fluid Phase Equil. 140, 233-244. 

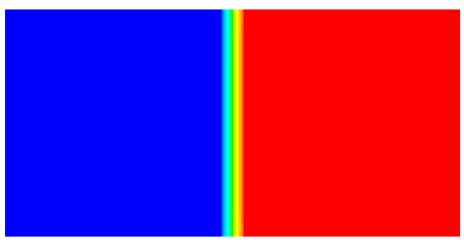

(a) $\operatorname{Re}=112$
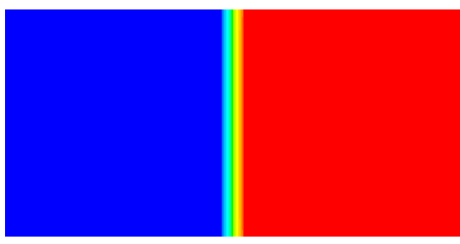

(c) $\operatorname{Re}=137$

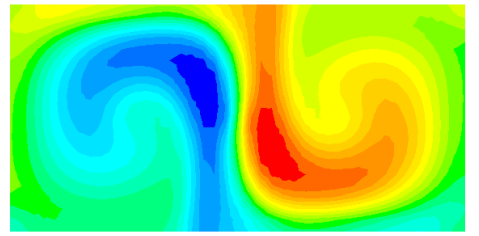

(e) $\operatorname{Re}=160$
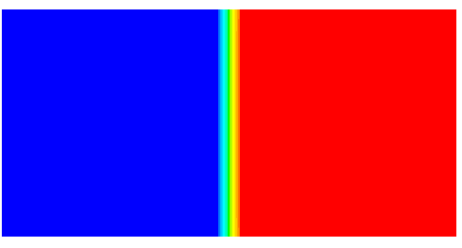

(b) $\operatorname{Re}=125$

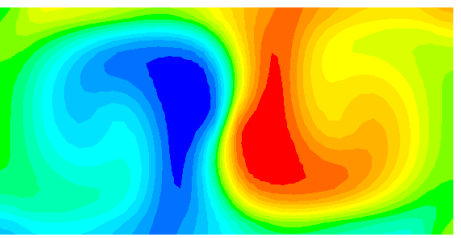

(d) $\mathrm{Re}=150$

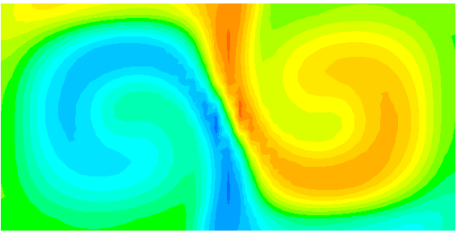

(f) $\operatorname{Re}=175$

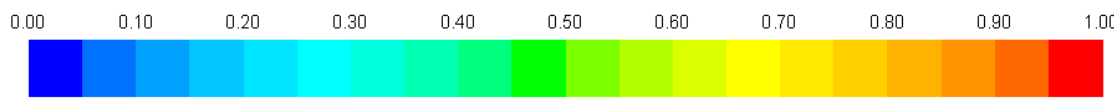

Figure 4: Concentration distribution in the mixing channel outlet section, i.e. $y=1.5 d$, for $\alpha=1$ 


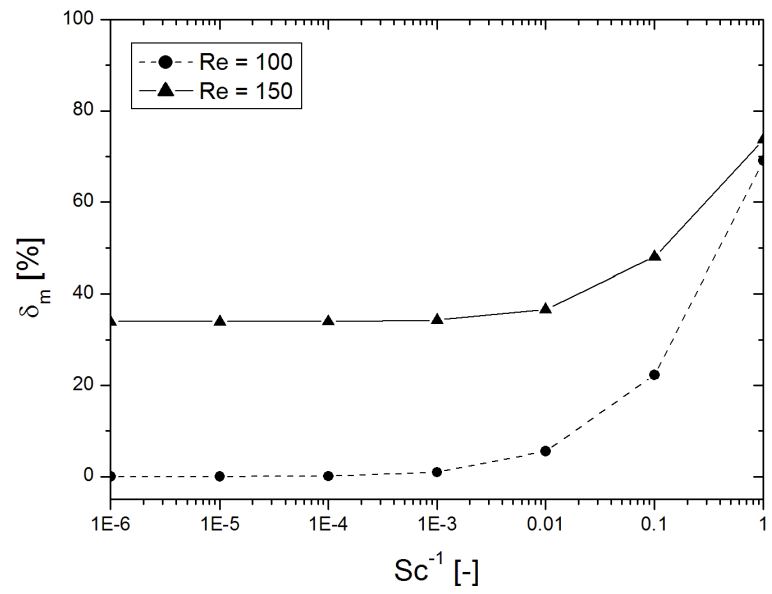

Figure 5: Degree of mixing as a function of the inverse of the Schmidt number for $R e=100$ and 150 .

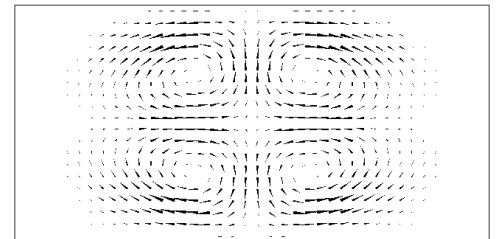

(a) $\operatorname{Re}=112$

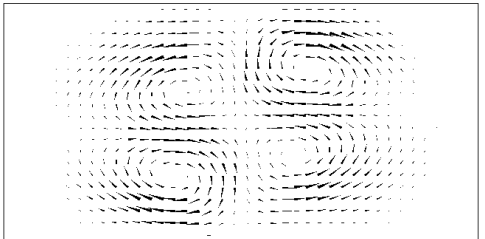

(c) $\operatorname{Re}=137$

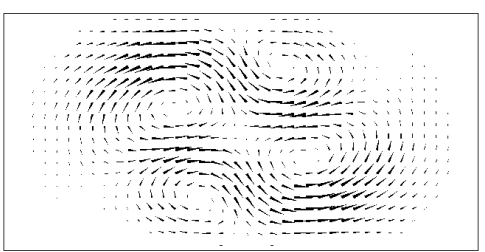

(e) $\operatorname{Re}=160$

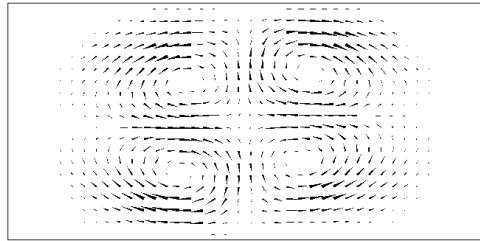

(b) $\operatorname{Re}=125$

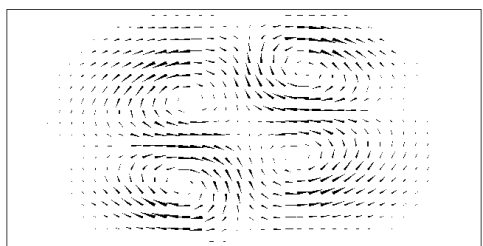

(d) $\operatorname{Re}=150$

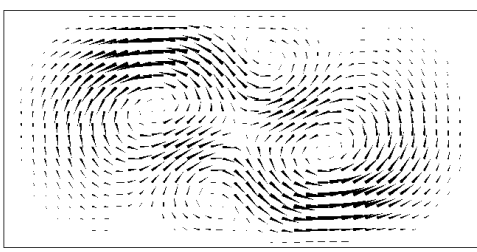

(f) $\operatorname{Re}=175$

Figure 6: Vector plots in the mixing channel cross section at $y=1.5 d$ for $\alpha=3$ at different Re numbers. 


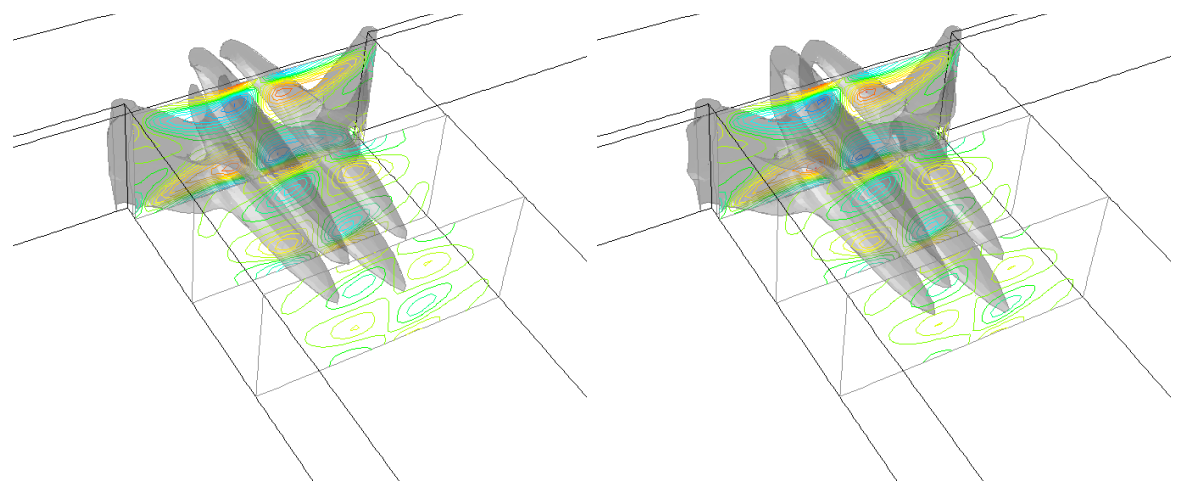

(a) $\operatorname{Re}=125$

(b) $\operatorname{Re}=137$

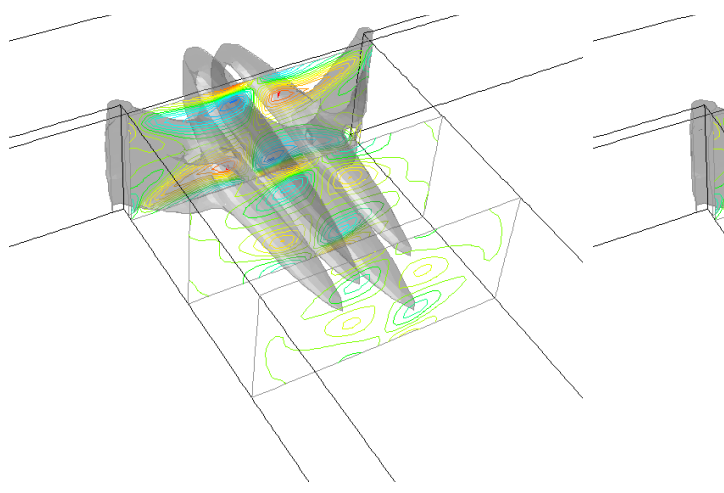

(c) $\operatorname{Re}=150$ (b) Re $=137$

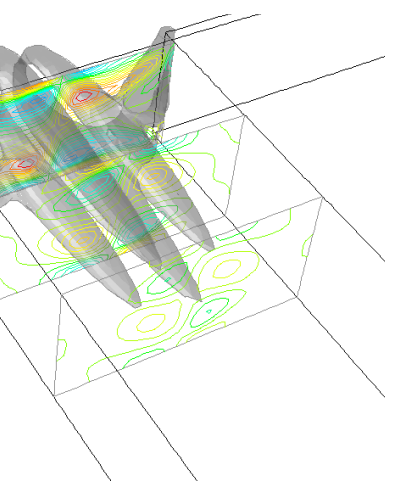

(d) $\operatorname{Re}=160$

Figure 7: Identified vortices according to the $\lambda_{2}$ criterion and distribution of normal vorticity for $\alpha=3$ and different $R e$ numbers at planes: $y=0.1 d, 0.8 d$ and $1.5 d$. 

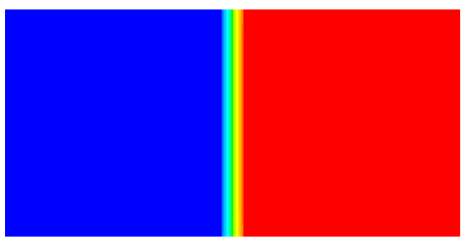

(a) $\operatorname{Re}=112$

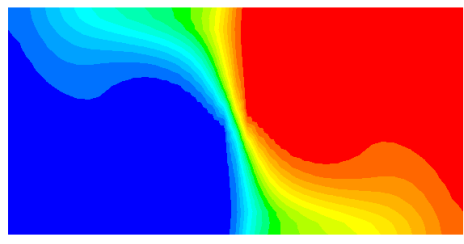

(c) $\operatorname{Re}=137$

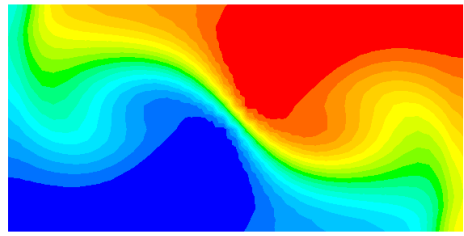

(e) $\operatorname{Re}=160$

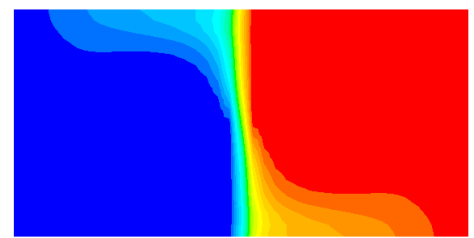

(b) $\operatorname{Re}=125$

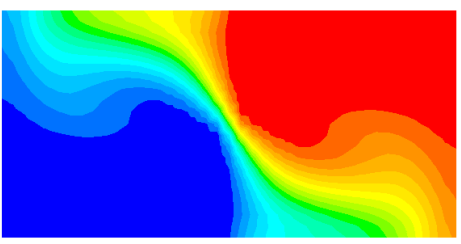

(d) $\operatorname{Re}=150$

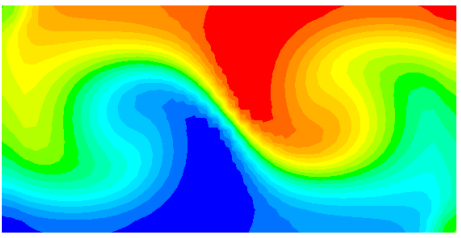

(f) $\operatorname{Re}=175$

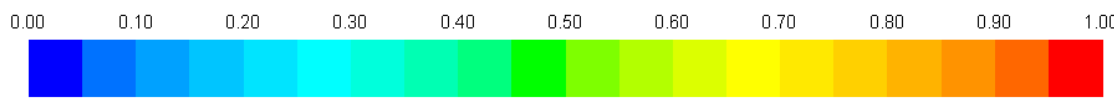

Figure 8: Concentration distribution in the mixing channel outlet section, i.e. $y=1.5 d$, for $\alpha=3$ 


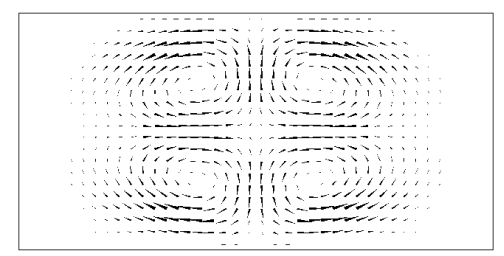

(a) $\operatorname{Re}=112$

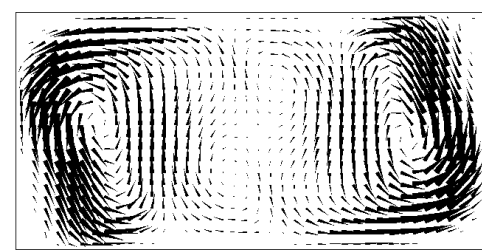

(c) $\operatorname{Re}=125$

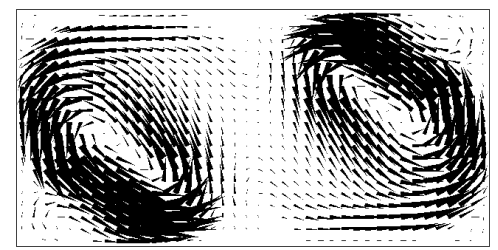

(e) $\operatorname{Re}=150$

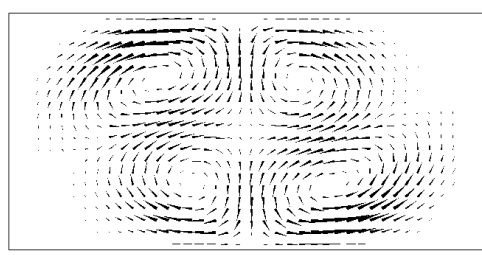

(b) $\operatorname{Re}=118$

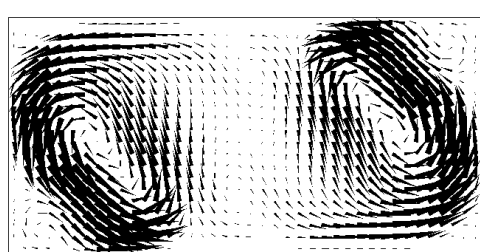

(d) $\mathrm{Re}=137$

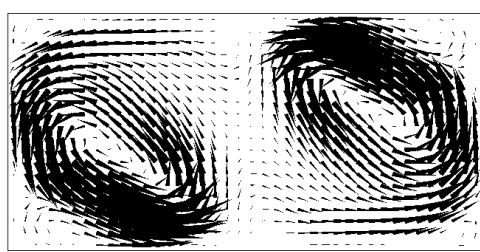

(f) $\operatorname{Re}=160$

Figure 9: Vector plots in the mixing channel cross section at $y=1.5 d$ for $\alpha=1 / 3$ at different Re numbers 


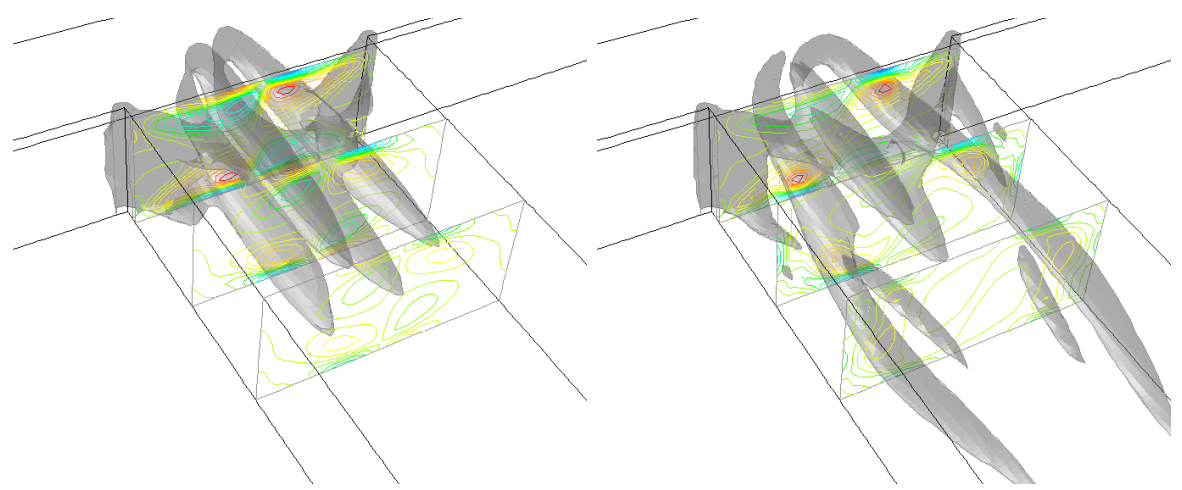

(a) $\operatorname{Re}=118$

(b) $\operatorname{Re}=125$

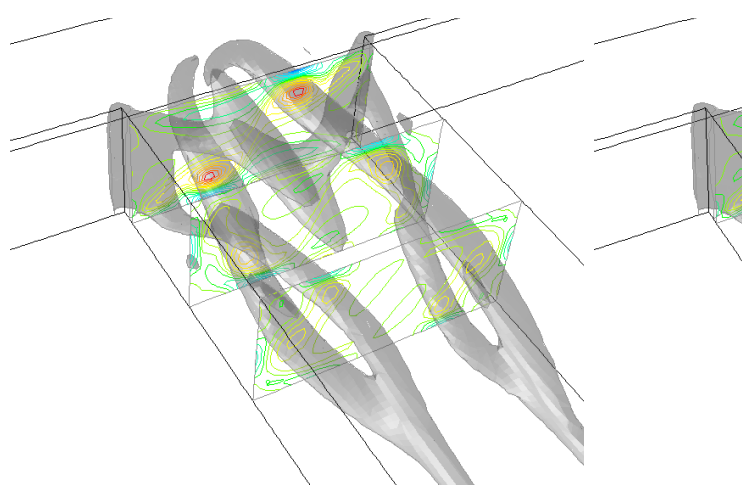

(c) $\operatorname{Re}=137$ (b)

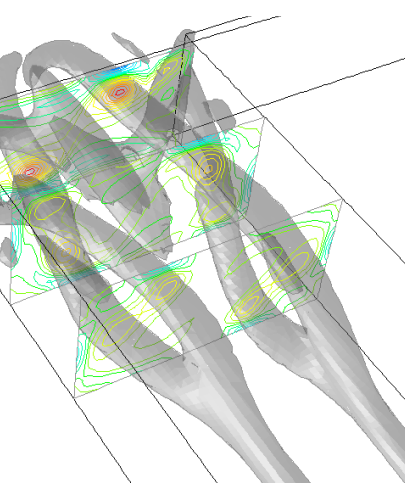

(d) $\operatorname{Re}=160$

Figure 10: Identified vortices according to the $\lambda_{2}$ criterion and distribution of normal vorticity for $\alpha=1 / 3$ and different Re numbers at planes: $y=0.1 d, 0.8 d$ and $1.5 d$. 


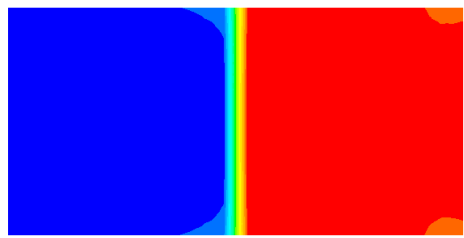

(a) $\operatorname{Re}=112$

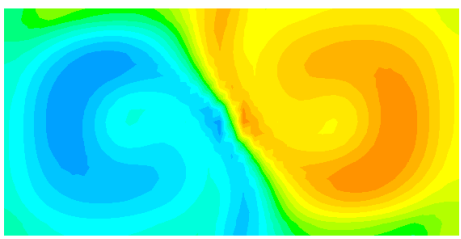

(c) $\operatorname{Re}=125$

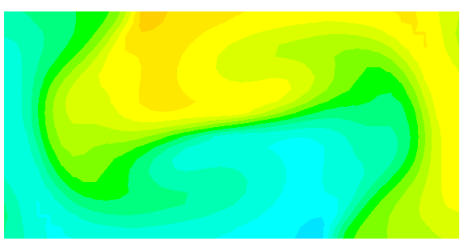

(e) $\operatorname{Re}=150$

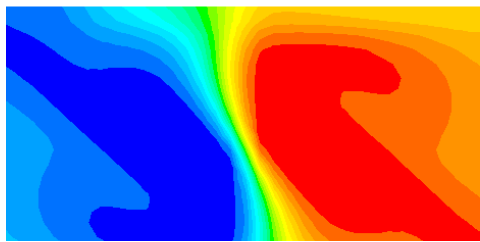

(b) $\operatorname{Re}=118$

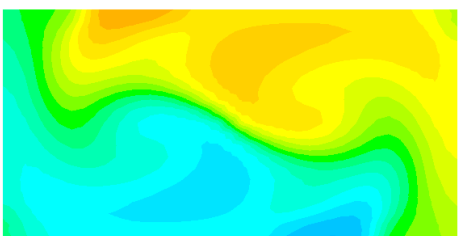

(d) $\operatorname{Re}=137$

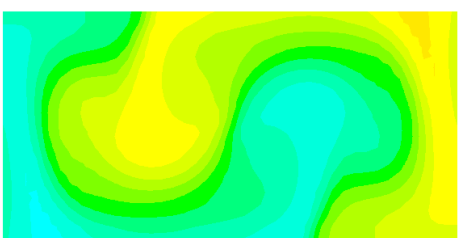

(f) $\operatorname{Re}=160$

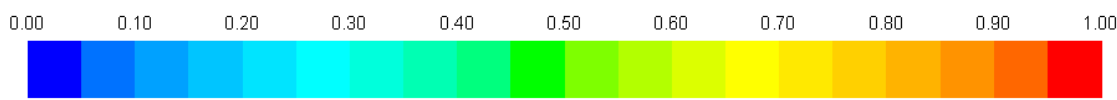

Figure 11: Concentration distribution in the mixing channel outlet section, i.e. $y=1.5 d$, for $\alpha=1 / 3$ 


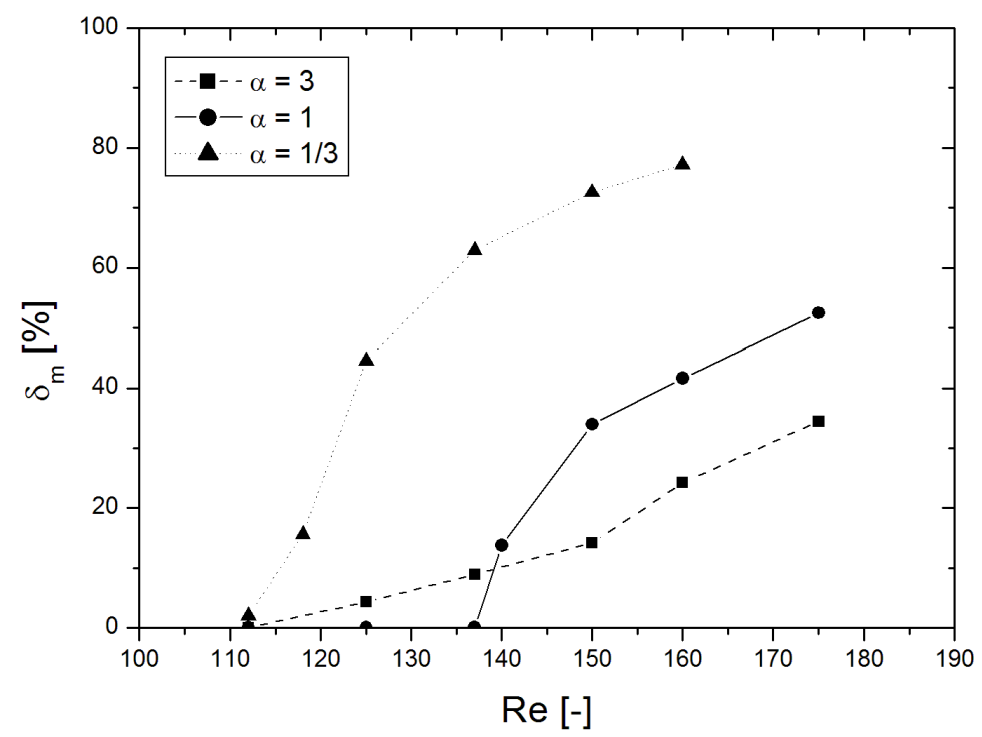

Figure 12: Degree of mixing for the three $\alpha$ cases as a function of the Re number 

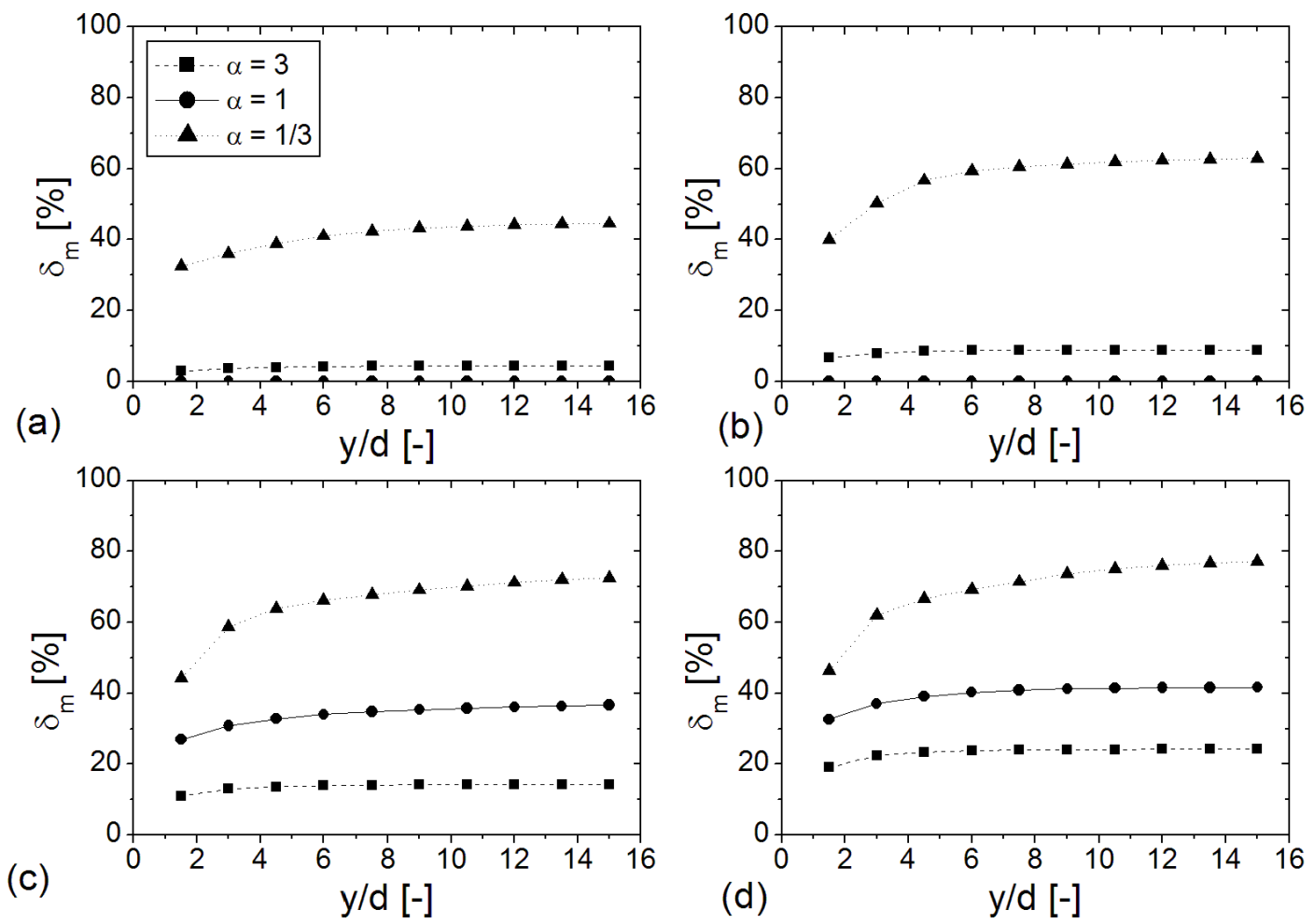

Figure 13: Degree of mixing along the channel for the three $\alpha$ cases at: (a) $R e=125$; (b) $R e=137 ;$ (c) $R e=150 ;$ (d) $R e=160$. 